\title{
REGENERACIÓN Y CAMBIOS DE DIVERSIDAD EN GRANDES INCENDIOS A PARTIR DE IMÁGENES SATÉLITE LANDSAT TM Y ETM+
}

\author{
R. HERNÁNDEZ CLEMENTE(1), R. M. NAVARRO CERRILLO(1) \\ J. E. HERNÁNDEZ-BERMEJO'(2), S. ESCUIN ROYO(1) \\ A. GARCÍA-FERRER PORRAS ${ }^{(3)}$, M. SÁNCHEZ DE LA ORDEN(3)
}

(1) Dpto. de Ingeniería Forestal (Universidad de Córdoba) Avda. Menéndez Pidal, s/n, Córdoba 14004

(2) Dpto. de Ciencias y Recursos Agrícolas y Forestales (Universidad de Córdoba)

(3) Dpto. de Ingeniería Gráfica e Ingeniería y Sistemas de Información Geográfica (Universidad de Córdoba)

Correo electrónico de contacto: g82heclr@uco.es

RESUMEN: Los incendios modifican la estructura del paisaje, contribuyendo a su homogeneización y promoviendo, por tanto, mayores riesgos de incendio con el tiempo. Lejos del falso paradigma que ofrecían los procesos de autosucesión en ecosistemas mediterráneos, este estudio ofrece un análisis diacrónico de las pautas seguidas espacial y temporalmente por las principales formaciones vegetales instauradas en el área afectada. El incendio, ocurrido en la sierra de Huétor (Granada) en 1993 fue evaluado a partir de la información recogida de los inventarios realizados en los años 2000 y 2005 e integrado a la información obtenida a partir de las imágenes Landsat TM y ETM+ de los años 1992, 1993, 2000 y 2004. Los resultados obtenidos permitieron realizar un análisis integral de la regeneración a largo plazo dentro de la zona afectada. La resolución temporal y espacial de las imágenes permitió realizar una delimitación precisa de la zona afectada, la descripción actual de las principales comunidades presentes en el área y el desarrollo de modelos para el seguimiento de la regeneración de la cobertura vegetal y la dinámica de cambio en diversidad. Los valores de cambio registrados en NDVI muestran alta correlación con los valores de cobertura de las especies más significativas en los procesos de regeneración. La teledetección y los SIG constituyen una herramienta eficaz para la evaluación de la regeneración del grado de cobertura vegetal y el estudio de la dinámica postincendio de la diversidad y, por tanto, se recomienda su introducción en el manejo y la restauración de espacios naturales.

ABSTRACT: The role played by direct regeneration in early post-fire years is well known in mediterranean plant communities. However, the effect of wildfires 
on medium and long-term vegetation dynamics needs a better understanding. Spatial and temporal post-fire vegetation patterns are fundamental to evaluate forest succession processes, forest structure and species diversity. Focussing on that need, this work presents an analysis of regeneration patterns and diversity dynamics after a fire which occurred in 1993, affecting around $7000 \mathrm{Ha}$ in southern Spain. Field data were recorded from permanent plots in 2000 and 2005. Remotely sensed data were obtained from Landsat ETM+ images of the years 1992, 1993, 2000 and 2004. Both sets of data, fieldwork and remotely sensed data, were analyzed and modulated separately through statistical and quantitative analysis, and then related on the basis of ecological relations. Based on those results, we have developed a stochastic model to predict medium-term forest dynamics. Forests in this area followed the auto-succession model in the early years. However, because of the history of disturbance in the area, it seems unlikely that forests will return to their pre-fire state. Change on NDVI was highly correlated with the changes recorded in the regrowth of the main species, which conditioned the composition of the plant species, and, therefore, diversity indexes such as Shannon $(H)$ and Simpson (D). As a general conclusion, it can be affirmed that NDVI was highlighted as a good understanding of succession stage changes, showing sensitivity to post-fire plant cover changes and diversity dynamics, which therefore recommends its application in the management and restoration of wildfire-affected areas.

Palabras clave: Detección de cambios, regeneración post-incendio, teledetección, NDVI, vegetación mediterránea.

Keywords: Fire ecology, diversity, succession patterns, remote sensing, NDVI, dynamic modelling.

Enviado el 20 de junio de 2007 Aceptado el 15 de octubre de 2007

\section{Introducción}

Independientemente de la variabilidad de los efectos del fuego en la vegetación, está ampliamente aceptado (Hanes, 1971; Trabaud y Lepart, 1980) que los ecosistemas mediterráneos afectados por el fuego presentan un modelo de regeneración directa o autosucesión. Por otra parte, estas comunidades vegetales presentan una alta resilencia a través de diferentes tipos de estrategias (Naveh, 1975; Pausas, 2004). No obstante, no todas las plantas mediterráneas sobreviven al fuego; de hecho, hay muchas que no han desarrollado ningún tipo de mecanismo de defensa o regeneración (Piussi, 1992), e incluso aquellas que lo poseen están condicionadas a innumerables factores ambientales que limitan su eficiencia. Por lo tanto, los modelos de regeneración de los ecosistemas mediterráneos son difíciles de configurar y requieren una amplia base de observaciones científicas, que son todavía insuficientes debido a la escala temporal tan amplia que requieren. En este sentido la información que proporcionan las imágenes de satélite juega un papel fundamental y el estudio de la regeneración ha sido una de las principa- 
les aplicaciones en el entorno del Mediterráneo (Viedma et al., 1997; Henry y Hope, 1998; Salvador et al., 2000; Riaño et al., 2002; Díaz-Delgado, 2001).

A pesar de la variedad de trabajos realizados sobre ecología de incendios, son escasos los estudios que integren la información remota y el análisis de la vegetación asociado a cambios en la biodiversidad; en particular, aquellos que estudian los cambios a la escala de comunidad (concretamente para matorrales), con suficiente apoyo de datos de campo y para intervalos largos de tiempo tras la presencia de un incendio forestal. La necesidad de llevar a cabo actuaciones de conservación y restauración de las zonas afectadas por incendios forestales obliga a tener un conocimiento adecuado de los recursos que en ellas existen y del sistema de relaciones naturales que actúan sobre ellas. Por ello, el objetivo de este trabajo fue evaluar el proceso multitemporal de cambio en variables de estructura y composición de matorrales mediterráneos afectados por un incendio. Para alcanzar dicho objetivo se analizaron parcelas situadas en el mismo punto geográfico en dos momentos distintos del período de regeneración. Comparando dichos datos se evaluaron los cambios post-incendio en composición específica y biomasa, con el fin de aproximarse a la evolución de la composición florística, examinando los procesos de competencia y capacidad de regeneración.

\section{Material y métodos}

\subsection{Zona de estudio}

El área de estudio está localizada en la hoja (1010) del MTN, 1:50.000 (coordenadas UTM: ULX: 457343; LRX: 473918; ULY: 4129353; LRY: 4118806) y fue afectada por un incendio ocurrido los días 7 y 10 de agosto de 1993 en la provincia de Granada (Sur de España) que afectó a más de 7.000 ha. Alrededor de 205 ha pertenecían al Parque Natural de la Sierra de Huétor. Las vías de comunicación más usuales con esta área parten de la A-92 y de la carretera comarcal GR-SE-39, que une a las localidades de La Peza y Quéntar.

La zona incendiada presenta una orografía accidentada con pendientes entre el 10\% y el $40 \%$ y altitudes comprendidas entre los 1.000 y los $1.700 \mathrm{~m}$. El complejo montañoso se encuentra enclavado climáticamente en la transición del mediterráneo seco al mediterráneo subhúmedo, con precipitaciones medias anuales de $600 \mathrm{~mm}$. De acuerdo con Rivas-Martínez et al. (1997), la zona de estudio está situada en el Sector Malacitano-Almijarense y climáticamente pertenece al piso supramediterráneo. La principal característica termométrica es la acusada continentalidad del espacio natural y sus zonas adyacentes, con diferencias de temperatura entre invierno y verano superiores a $15^{\circ} \mathrm{C}$, tanto entre máximas como entre mínimas.

La litología se caracteriza principalmente por sustratos básicos como calizas, calizas marmóreas y margas y en menor medida por esquistos, gneises y pizarras, que dan lugar a suelos ácidos. Según la Soil Taxonomy (USDA) los suelos son esqueléticos (lito- 
soles y regosoles) y poco desarrollados debido a la dureza de la roca originaria (caliza) $\mathrm{y}$ a las altas pendientes en las que se desarrolla.

La vegetación de la zona ha sido fuertemente modificada por la acción del hombre que ha producido una degradación muy avanzada. En la década de los 40 comenzó una intensa actividad repobladora que utilizó Pinus pinaster Aiton, Pinus halepensis Miller, Pinus nigra Arnold y en menor medida Pinus sylvestris L. Tras el incendio de 1993, han sido escasas las labores de reforestación desarrolladas en la zona. La primera, a finales de 1996, consistió en una siembra aérea con muy bajos resultados. Las siguientes actuaciones, plantando directamente sobre terreno con ayuda de maquinaria, han sido puntuales y con escasa relevancia. A partir de las series de vegetación potencial de la zona de estudio y el conocimiento del estado actual, pueden definirse las siguientes series de vegetación dominantes:

1. Encinares mesomediterráneos béticos y basófilos que llevarían como especies dominantes Quercus ilex subsp. ballota (Desf.) Samp.(= Q. rotundifolia Lam), Juniperus oxycedrus L. y Crataegus monogyna subsp. brevispina (Kunze \& Franco), bosque cuya degradación produce aulagares con Ulex parviflorus Pourret, Rosmarinus officinalis L., Cistus clusii Dunal, Lavandula lanata Boiss. y Daphne gnidium L.

2. Encinares supramediterráneos béticos y basófilos con Quercus ilex como dominante, a veces transformados en pinares de Pinus pinaster.

3. Supramediterránea silicícola de la encina y el quejigo, generalmente transformados en pinares de pino negral, con presencia a veces de Quercus faginea Lam. y abundancia de Adenocarpus decorticans Boiss.

\subsection{Datos de campo}

Se realizaron dos campañas de campo, la primera en agosto del año 2000 y otra posterior en el 2005. En ambos casos se aplicó la misma metodología de muestreo y las repeticiones realizadas en el 2005 se realizaron en los mismos puntos geográficos que en la campaña anterior. En el 2000 se seleccionaron un total de 33 parcelas a partir de una estratificación de la vegetación mediante el análisis visual de imágenes Indian Remote Sensing Pancromática (IRS-PAN) de 1998, una fotografía aérea (1:20.000) de 1999 y mapas topográficos (1:10.000), delimitando las teselas de vegetación de diferente textura y tono. En el año 2005 se seleccionaron un número de parcelas representativo de cada agrupación derivada de un análisis estadístico de cluster, el cual fue obtenido a partir de la fracción de cobertura por especie inventariada en el año 2000. En total se inventariaron 15 de las 33 iniciales (Hernández et al., 2006).

Las parcelas se localizaron en campo a partir de la cartografía 1:10.000 con coordenadas reales UTM y un GPS (error $<10 \mathrm{~m}$ ) para el año 2000 y un GPS (Magellan Meridian Color) (error $<3 \mathrm{~m}$ ) en el año 2005. Se estima un error medio de replanteo inferior 
a 3-5 m., que se considera no distorsiona significativamente el análisis debido a la homogeneidad de las teselas seleccionadas.

Con apoyo de una cinta métrica se replantearon las parcelas de $50 \mathrm{~m}$ de diámetro definiendo dos transectos perpendiculares entre sí, siguiendo uno de ellos la dirección de la máxima pendiente y partiendo del centro de cada parcela. La superficie total muestreada fue de $29.452,5 \mathrm{~m}^{2}$.

El método utilizado para la realización de los inventarios fue el método de intercepción lineal (Bonham, 1989). Se inventariaron separadamente los doseles arbóreo y de matorral. En este último se incluyeron además de las especies arbustivas presentes, el regenerado de especies arbóreas y especies herbáceas perennes. En cada par de transectos se tomaron datos de diámetro proyectado de copa " $\varnothing^{p "(m) ~ p a r a ~ e l ~ d o s e l ~ a r b o ́ r e o ~ y ~}$ longitud.

A partir de estos datos se estimó la cobertura y la diversidad florística. Para ello se calcularon las siguientes variables:

- Cálculo de cobertura vegetal $\left(\mathrm{C}_{\mathrm{di}}\right)$, se obtuvo como sumatoria de la fracción de cobertura interceptada por cada especie $(\mathrm{k})$. para el dosel de matorral $\mathrm{C}_{\mathrm{dm}}$ y para el dosel arbóreo $\mathrm{C}_{\mathrm{d} a}$ :

$$
\begin{aligned}
& C_{d m}=\Sigma l_{k} / 100, \text { expresado en }\left(\mathrm{m}^{2} / \mathrm{m}^{2}\right) \\
& \mathrm{C}_{\mathrm{da}}=\Sigma \emptyset_{\mathrm{k}}^{\mathrm{p}} / 100, \text { expresado en }\left(\mathrm{m}^{2} / \mathrm{m}^{2}\right)
\end{aligned}
$$

siendo $l_{k}$ la fracción de matorral interceptado y $\emptyset_{k}^{p}$ proyección de copa interceptada.

- Riqueza (S) o número total de especies diferentes. Mide la heterogeneidad de la vegetación y proporciona información de la composición florística de la comunidad.

- Diversidad :

Índice de Simpson: $(D)=1 / \Sigma p_{i}^{2}$

Índice de Shannon-Wiener: $(H)=-\Sigma p_{i} \ln p_{i}$

donde $p_{i}=n_{i} / N$, siendo $n_{i}$ el número de individuos de la especie ' $i$ ' y $N$ la abundancia total de las especies.

\subsection{Procesamiento de las imágenes}

En la Tabla 1 se muestra la selección de imágenes empleadas para el análisis multitemporal. Todas las imágenes fueron corregidas geométricamente usando una trans- 
formación polinomial de primer orden, obtenida a partir de puntos de control (Ground Control Points, GCPs). Dichos puntos fueron localizados sobre una ortofoto del año 2000 y 0,5 m. de resolución, obteniéndose un error medio cuadrático inferior a 1 píxel. La transformación de los niveles digitales a radiancia se llevó a cabo a partir de los coeficientes de calibración estándar de ambos sensores. A partir de estos valores de radiancia se calcularon los valores de reflectividad aparente y se realizó una normalización final para homogeneizar el error producido por la atmósfera a partir de áreas invariantes (Caselles et al., 1989), obteniéndose un coeficiente de correlación de 0,98 y un error estándar de 0,029. Una vez corregidas las imágenes se calculó el índice normalizado de vegetación (NDVI) obtenido a partir de las bandas 3 y 4 de cada una de las imágenes.

Tabla 1. Relación de imágenes Landsat utilizadas en el estudio de cambios de vegetación del incendio de sierra de Huétor (Granada)

\begin{tabular}{|l|c|c|c|c|}
\hline Fase & Satélite & Escena & $\begin{array}{c}\text { Ángulo de } \\
\text { elevación }\end{array}$ & Azimuth \\
\hline Anterior al incendio & Landsat 7 & ETM+ 17/08/92-200-34 & 53,27 & 122,96 \\
\hline Posterior al incendio & Landsat 7 & ETM+ 05/09/93-200-34-b & 49,26 & 130,97 \\
\hline $\begin{array}{l}\text { Campaña de } \\
\text { inventarios 2000 }\end{array}$ & Landsat 7 & ETM+ 31/08/00-200-34 & 54,29 & 138,56 \\
\hline $\begin{array}{l}\text { Campaña de } \\
\text { inventarios 2005 }\end{array}$ & Landsat 5 & TM 18/08/04-200-34 & 60,18 & 139,90 \\
\hline
\end{tabular}

\subsection{Proceso metodológico}

\subsubsection{Delimitación de la zona afectada}

La determinación del perímetro se llevó a cabo aplicando un análisis de componentes principales (ACP) (Siljestrom y Moreno, 1995; Heredia et al., 2003). Este método consistió en realizar las componentes principales $(\mathrm{CP})$ sobre la imagen compuesta de 8 bandas $(3,4,5$ y 7) del año 93 y del año 92. Se calcularon el mismo número de componentes principales que de bandas se disponía para el análisis (8). Generalmente los primeros $\mathrm{CP}$ son los que absorben la mayor proporción de varianza y representan el sustrato común entre fechas. Los $\mathrm{CP}$ residuales reflejan, por el contrario, el cambio registrado en ese intervalo de tiempo. Cuando el cambio es producido por un incendio forestal, se elige como componente de cambio aquella que muestre una alteración en el signo de los vectores propios asociados a la banda 4 , debido al efecto que produce el fuego sobre las coberturas vegetales. Una vez seleccionado el componente principal con mayor poder de discriminación se obtuvo la cartografía del área quemada. 


\subsubsection{Tipificación de la vegetación}

Los tipos de vegetación se clasificaron a partir de las bandas 3, 4 y 5 de la imagen Landsat ETM+ del año 2005. Estas bandas fueron seleccionadas en base a la relación de proximidad derivada de una clasificación no supervisada realizada previamente sobre la misma imagen. El criterio elegido para la asignación categórica de los píxeles fue el de mínima distancia espectral, tomándose un total de 160 puntos de entrenamiento en campo (20 por categoría), intentando abarcar las variaciones espaciales que se presentaban en cada categoría, como orientación, pendiente, densidad o tipo de suelo. A partir de dichos puntos y el conocimiento del área, se tomaron hasta un total de 2.004 píxeles (aproximadamente 200 por clase) sobre la ortofoto del año 2002 con resolución de $0,5 \mathrm{~m}$.

\subsubsection{Modelización de la regeneración de la cobertura vegetal}

Los modelos de regeneración fueron obtenidos a partir de la correlación de los valores de cobertura recogidos en los inventarios realizados en el año 2000 y 2005 y los valores de NDVI relativos a las imágenes de las respectivas fechas. Con una matriz de 3 x 3 se extrajeron los valores relativos a ambas imágenes sobre los puntos de muestreo. Con los datos obtenidos se generaron diferentes modelos de regresión, seleccionándose aquél que mejor se ajustara a la regeneración de la cubierta vegetal. Contrastado y validado el modelo en ambos períodos, se aplicó el más reciente para evaluar el grado de cobertura previa al incendio. Dicho criterio se asumió debido a la ausencia de datos relativos al año anterior al incendio.

\subsubsection{Modelización del cambio en diversidad y evolución de las especies dominantes}

El objetivo de este análisis fue discernir las pautas de cambio de la diversidad establecidas en el área incendiada en los últimos cinco años, de tal forma que se posibilite la evaluación de los procesos de sucesión. Para ello se realizó un análisis de correlación entre las variables estimadas de cambio por parcela en diversidad, cobertura por especie indicadora y dominante y valor de NDVI. Se consideraron especies indicadoras aquellas que resultaron significativas al cambio a través de un análisis de la varianza con medidas repetidas realizada a partir de las coberturas inventariadas en el año 2000 y el año 2005. Un mayor detalle de dichos análisis puede encontrarse en Hernández-Clemente et al. (2006). Se consideraron especies dominantes aquellas que resultaron cuantitativamente dominantes en el período analizado.

El cambio asociado a dichas variables se obtuvo como diferencia media del año 2005 menos la media del año 2000 por parcela y se calcularon a partir de los valores de:

- Índices de diversidad (Índice de Shannon-Wiener, H, Índice de Simpson, D y riqueza, $S$ ).

- Cobertura lineal total $(\%)$.

- Cobertura lineal de las especies indicadoras (\%). 
- Cobertura lineal de las especies dominantes (\%).

- NDVI, obtenido a partir de la diferencia:

$$
\text { DIFNDVI }[t 2-t 1]=N D V I i j\left[t_{2}\right]-N D V I_{i j}\left[t_{1}\right]
$$

donde $i j$ : coordenada del píxel, $t_{2}=2005$ y $t_{1}=2000$.

Los coeficientes de correlación estimados fueron los de Pearson, Tau-b de Kendall y Spearman. Todos varían entre -1 y 1 , indicando con esto la relación negativa o positiva, respectivamente, y toma un valor de 0 cuando la relación lineal es nula.

El número final de parcelas introducidas en el análisis de correlación fue de 10 , todas compuestas exclusivamente de plantas con porte arbustivo. Dicha selección se realizó a partir de un análisis de conglomerados jerárquico previo, de manera que las parcelas analizadas cubrieran de forma representativa todo el abanico florístico inventariado en la zona de estudio.

\section{Resultados y discusión}

\subsection{Delimitación de la zona afectada}

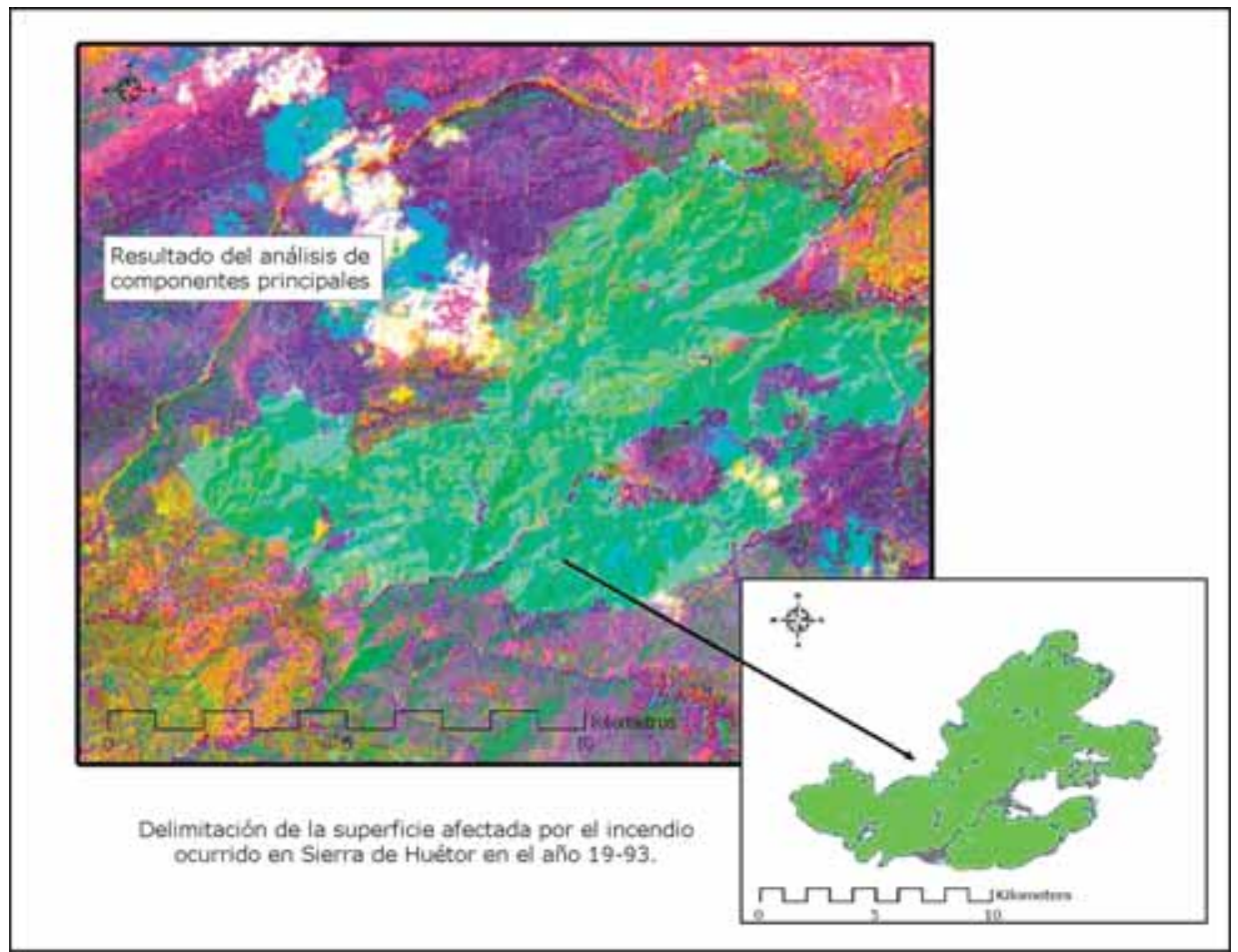

Figura 1. Cartografía del área afectada por el incendio que asoló la sierra de Huétor, Granada, en el año 1993. Método: Análisis de Componentes Principales 
La componente principal 5 fue la más adecuada para la determinación del área incendiada, mostrando una inversión completa de signos del año 2000 al 2005. A partir de estos resultados se extrajo la cartografía final del área afectada que aparece en la Figura 1. El área obtenida con GPS fue de 7525,98 ha y el área obtenida a partir de la imagen fue de 6948,63 ha, eso reflejaba una diferencia de 551,36 ha de áreas interiores no quemadas, identificables sólo con ayuda de la información remota, superando otros métodos tradicionales (fotointerpretación o medición del perímetro sobre el terreno) (Díaz-Delgado y Pons, 2001).

La validación en campo de la cartografía resultante mostraba valores de fiabilidad de $96,77 \%$ para el usuario y de $96,55 \%$ para el productor.

\subsection{Tipificación de la vegetación}

En la Figura 2 se muestra el resultado de la clasificación supervisada obtenida a partir de la imagen Landsat 5 del año 2005. Las clases con mayor separabilidad eran las clases con y sin vegetación. Dentro de la vegetación, las clases con mayor proximidad y, por tanto, mayor riesgo de confusión en la clasificación corresponden a las clases aulagar-romeral, romeral-aulagar y encinar. Esto se debe a la alta heterogeneidad del paisaje a nivel de píxel, que en su conjunto muestra un valor único resultado de la mezcla espectral que presenta el mosaico de vegetación. No obstante, la diferencia espectral fue lo suficientemente significativa como para aproximar una cartografía acertada atendiendo a la dominancia en cobertura de dichas especies. Por último, la clase pinar mostró unos valores aceptables de distancia entre clases.

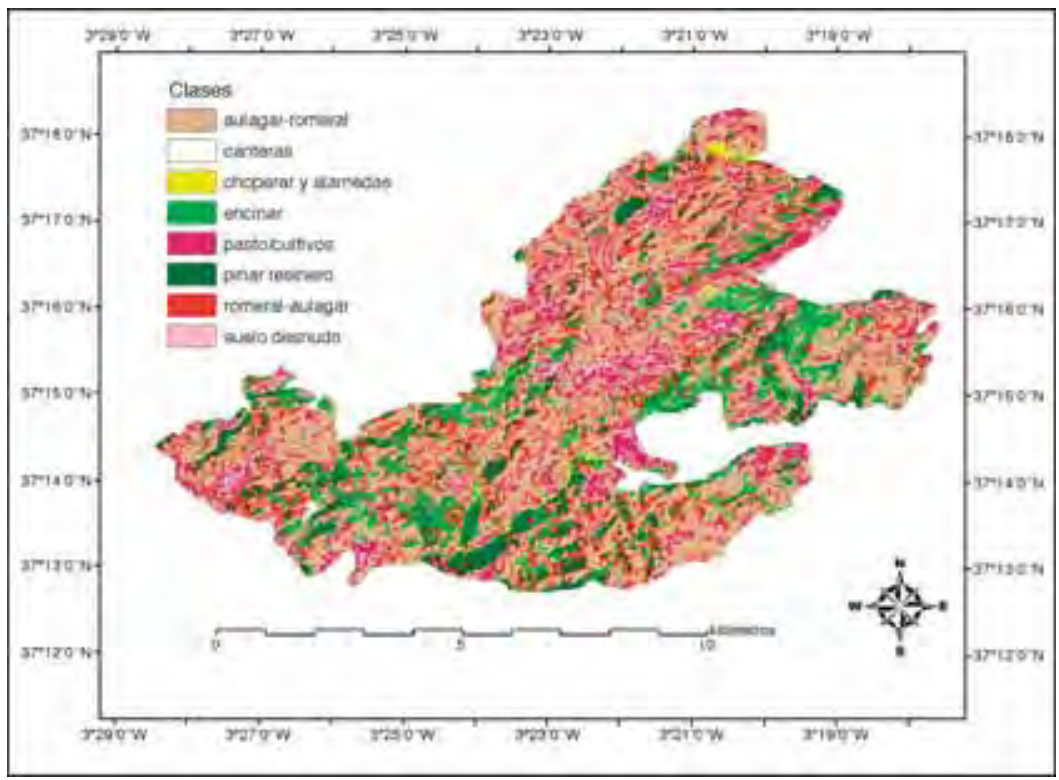

Figura 2. Clasificación supervisada obtenida a partir de la imagen Landsat 5 TM del año 2005 de los tipos de vegetación actuales dentro del área incendiada 
La fiabilidad global obtenida en la clasificación supervisada de los tipos de vegetación fue de un 84,5\% con un coeficiente Kappa de 0,78, siendo la fiabilidad total del productor del $83 \%$ y la del usuario del $86 \%$.

\subsection{Modelización de la regeneración de la cobertura vegetal}

El modelo que mejor ajustaba la estimación de cobertura vegetal fue de tipo lineal $\mathrm{Y}=\mathrm{a}+\mathrm{b} * \mathrm{X}$. El modelo así definido, se aplicó a las 33 parcelas inventariadas en el año 2000 y a las 18 inventariadas 5 años después (15 parcelas mas 3 parcelas de suelo desnudo). La reducción del número de muestreo es producto de la selección de las parcelas más características de los principales grupos de vegetación, derivada de un análisis cluster previo. Como paso intermedio, se validó el primero de los modelos reduciendo también el tamaño de muestra a 18, comprobando que los cambios eran poco significativos (Figura 3). La igualdad de los modelos [1] y [2] implica a su vez, que la ecuación característica deducida a partir de los valores de NDVI de la vegetación en proceso de autosucesión, transcurridos 7 años después de un incendio, se mantiene invariante en un período de 5 años.

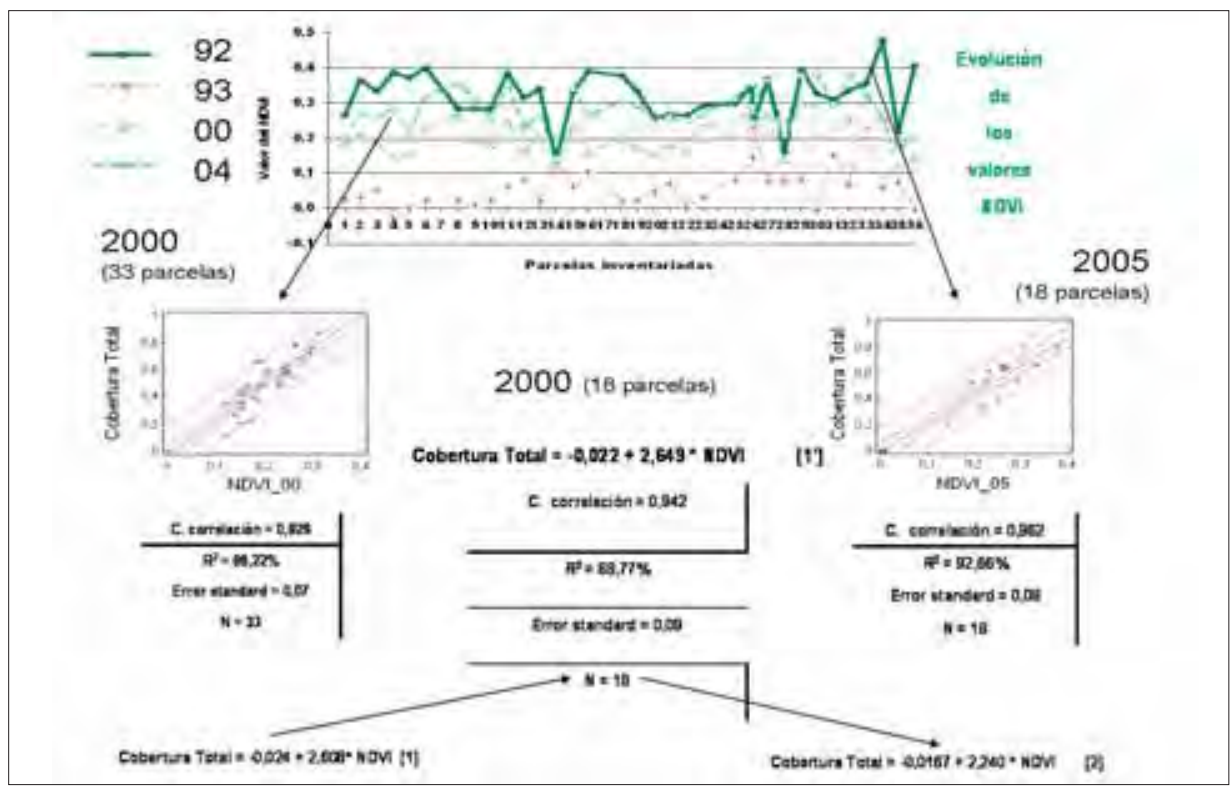

Figura 3. Regresión lineal de la cobertura por parcela y el valor de NDVI de las parcelas inventariadas en el año 2000 y el año 2005. Tamaños de muestra (N)

Las cartografías derivadas de estos modelos se muestran en la Figura 4. En 1992 el recubrimiento medio de niveles altos de cobertura vegetal (50-100\%) representaba un $63 \%$ del área. Tras el incendio, el porcentaje de regeneración para dichas clases alcanzaba un 38\% en el año 2000 y hasta un 52\% en 2005 (Tabla 2). En los resultados obtenidos para el año 1992 ha de asumirse un margen de error debido a la carencia de datos 
de campo previos al incendio. No obstante, en el año 2005 se identificaron en campo todos los grupos de vegetación existentes potencialmente en el área. Por ello se considera que la respuesta espectral de los principales grupos de vegetación existentes antes del incendio quedaría recogida en el modelo obtenido para dicho año.

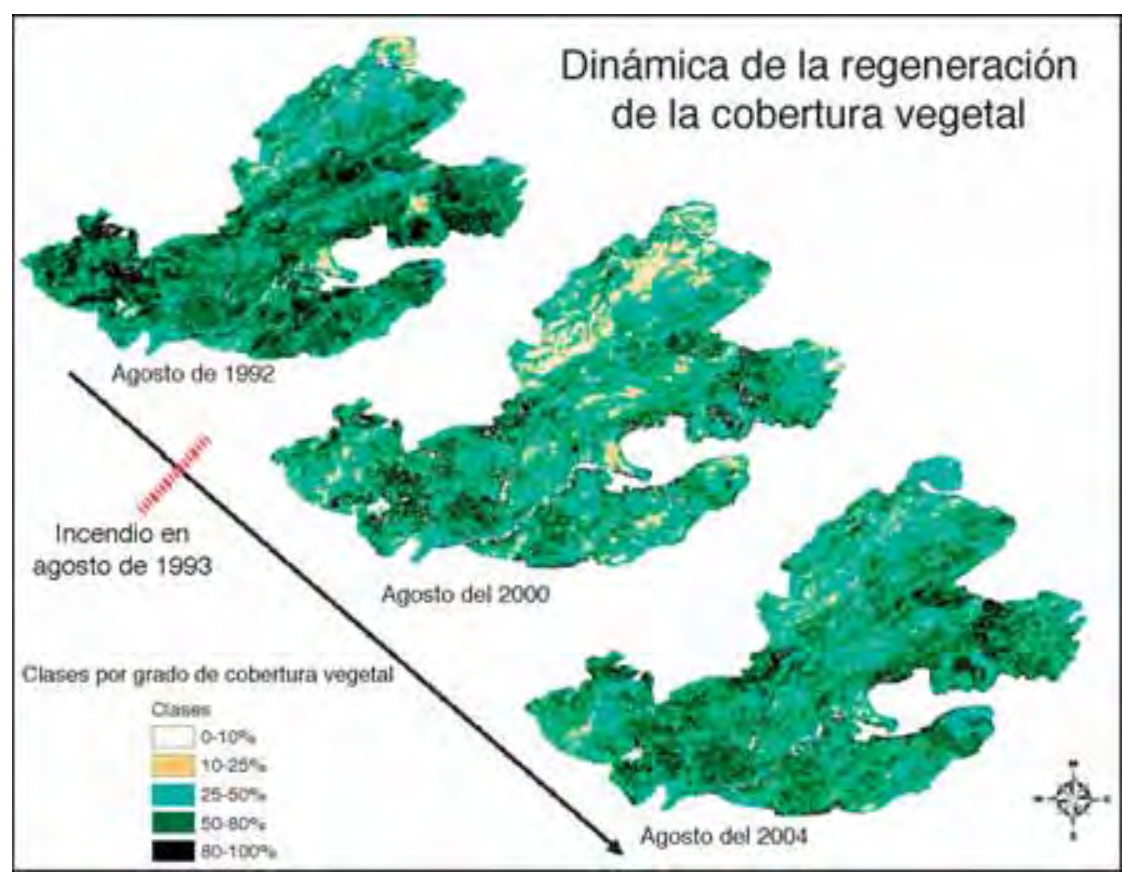

Figura 4. Clasificación supervisada del grado de cobertura vegetal dentro del perímetro del incendio a lo largo del tiempo (1992, 2000 y 2005)

Por último, es interesante destacar que la clase de cobertura más alta (80-100\%), relacionada con parcelas de arbolado denso, es la que peor tasa de recuperación registra.

Tabla 2. Relación de superficie relativa a la supervisada del grado de cobertura vegetal dentro del perímetro del incendio a lo largo del tiempo (1992, 2000 y 2005)

\begin{tabular}{|c|c|c|c|}
\hline Cobertura\% & NDVI_92 $(\%)$ & NDVI_00 $(\%)$ & NDVI_05 $(\%)$ \\
\hline $0-10$ & 0,79 & 4,52 & 1,41 \\
\hline $10-25$ & 3,26 & 13,26 & 4,37 \\
\hline $25-50$ & 32,48 & 43,76 & 40,94 \\
\hline $50-80$ & 51,38 & 32,25 & 46,85 \\
\hline $80-100$ & 12,09 & 6,20 & 6,43 \\
\hline
\end{tabular}


La fiabilidad global obtenida en el año 2000 fue de un 80,5\%, con un coeficiente Kappa de 0,74 , siendo la fiabilidad total del productor de un $79 \%$ y la del usuario de un $82 \%$.

\subsection{Modelización del cambio en diversidad y evolución de las especies dominantes}

La correlación establecida entre las variables de diversidad, cobertura asociada a las principales especies responsables del cambio y los valores de NDVI, resultó muy significativa y mostró un alto poder de discriminación de los patrones de cambio de la vegetación establecida en el área doce años después del incendio (Tabla 3). La diversidad (H, D) está altamente correlacionada y de forma positiva con la riqueza (S), lo cual marca una tendencia de incremento en el tiempo del número de especies por parcela. Los índices (H, D y S) están muy correlacionados y de forma negativa con la diferencia de la media de las coberturas de las especies dominantes, lo cual implica que la dinámica de regeneración está ligada directamente con las pautas de crecimiento de las especies dominantes: Ulex parviflorus, Rosmarinus officinalis y Cistus clusi. El NDVI muestra correlaciones significativas de forma positiva para los valores de cobertura vegetal (especialmente para la especie dominante Ulex parviflorus) y de forma negativa para las variables que miden la riqueza y la diversidad (especialmente para $\mathrm{S}$ ).

Es decir, doce años después del incendio, las especies dominantes (principalmente Ulex parviflorus) están produciendo un estancamiento de los procesos de sucesión secundaria, condicionando la introducción de nuevas especies y, con ello, el incremento de los valores de diversidad estimados a través de los índices de Shannon $(\mathrm{H})$, de Simpson (D) y el valor de la riqueza. Los valores de cambio en cobertura relacionados con la aulaga, en sus últimas fases del ciclo de senescencia, muestran directamente correlacionados con los valores de cambio de NDVI e inversamente correlacionados con los índices de diversidad. Los modelos obtenidos fueron integrados en un Sistema de Información Geográfica con el que se elaboró una cartografía semiautomática para la evaluación dinámica de la diversidad (Figuras 5 y 6).

Tabla 3. Análisis de correlación establecida entre las variables de diversidad $(D, H, S)$ y cobertura asociada a las principales especies responsables del cambio (Ulex parviflorus, Rosmarinus officinalis y Cistus clusi) (DifURC), cobertura de la especie dominante (Ulex parviflorus) (Dif_U), cobertura total (Dif_T) y los valores de NDVI (Dif-NDVI)

\begin{tabular}{|l|c|c|c|c|c|c|c|}
\cline { 2 - 8 } \multicolumn{1}{c|}{} & Dif_D & Dif_H & Dif_S & Dif_NDVI & Dif_URC & Dif_U & Dif_T \\
\hline Dif_D & 1,000 & $0,957 * * *$ & $0,699 *$ & $-0,748^{*}$ & $-0,816^{* *}$ & $-0,832 * *$ & $-0,578$ \\
\hline Dif_H & $0,957 * * *$ & 1,000 & $0,858 * *$ & $-0,842 * *$ & $-0,910 * * *$ & $-0,930 * * *$ & $-0,583$ \\
\hline Dif_S & $0,699 *$ & $0,858^{* *}$ & 1,000 & $-0,887 * * *$ & $-0,928 * * *$ & $-0,892 * * *$ & $-0,580$ \\
\hline Dif_NDVI & $-0,748^{*}$ & $-0,842 * *$ & $-0,887 * * *$ & 1,000 & $0,848 * *$ & $0,936 * * *$ & $0,713 *$ \\
\hline Dif_URC & $-0,816^{* *}$ & $-0,910 * * *$ & $-0,928 * * *$ & $0,848^{* *}$ & 1,000 & $0,884 * *$ & $0,694 *$ \\
\hline Dif_U & $-0,832 * *$ & $-0,930 * * *$ & $-0,892 * * *$ & $0,936 * * *$ & $0,884 * *$ & 1,000 & $0,712 *$ \\
\hline Dif_T & $-0,578$ & $-0,583$ & $-0,580$ & $0,713 *$ & 0,694 & 0,712 & 1,000 \\
\hline
\end{tabular}




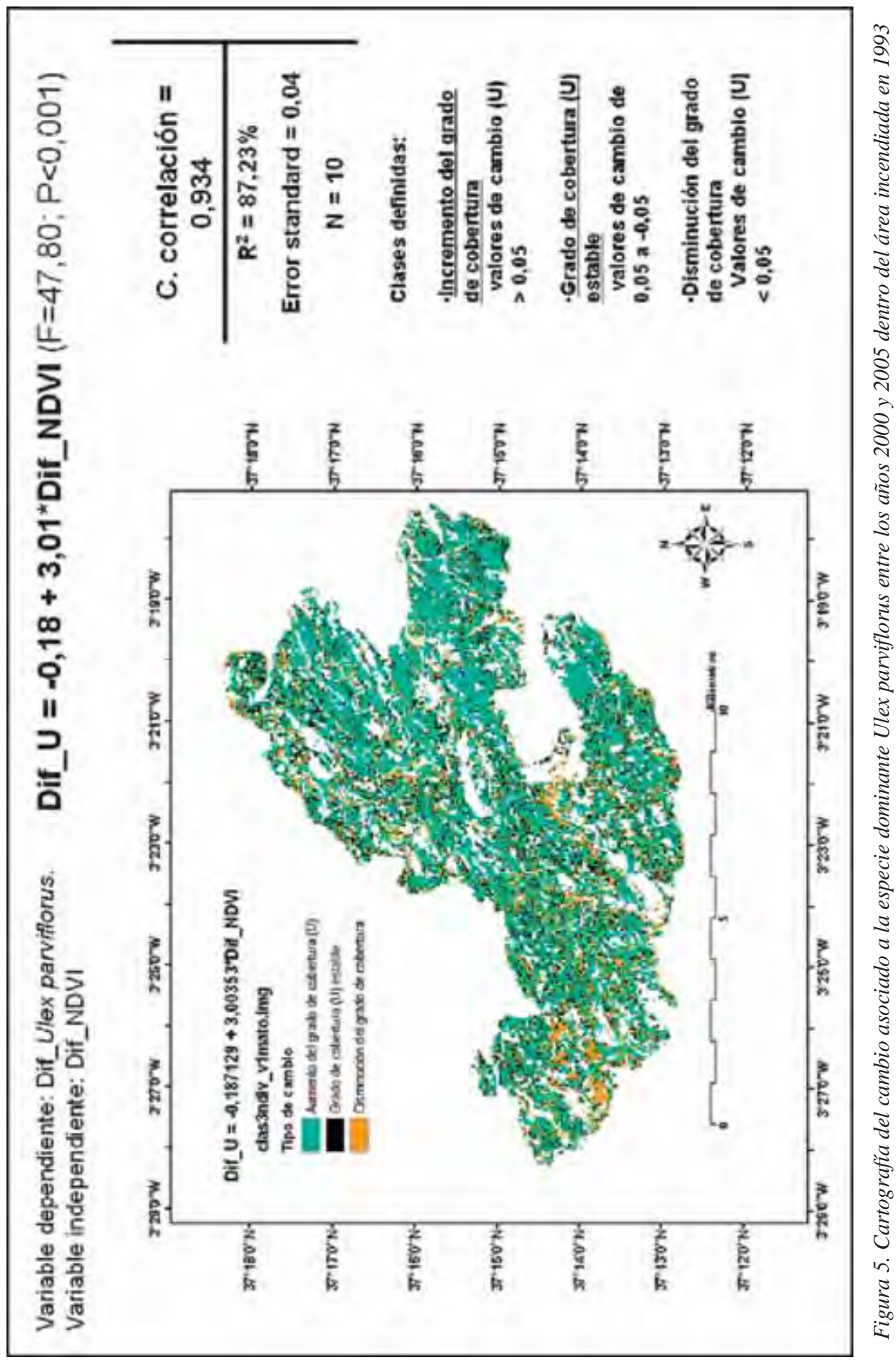




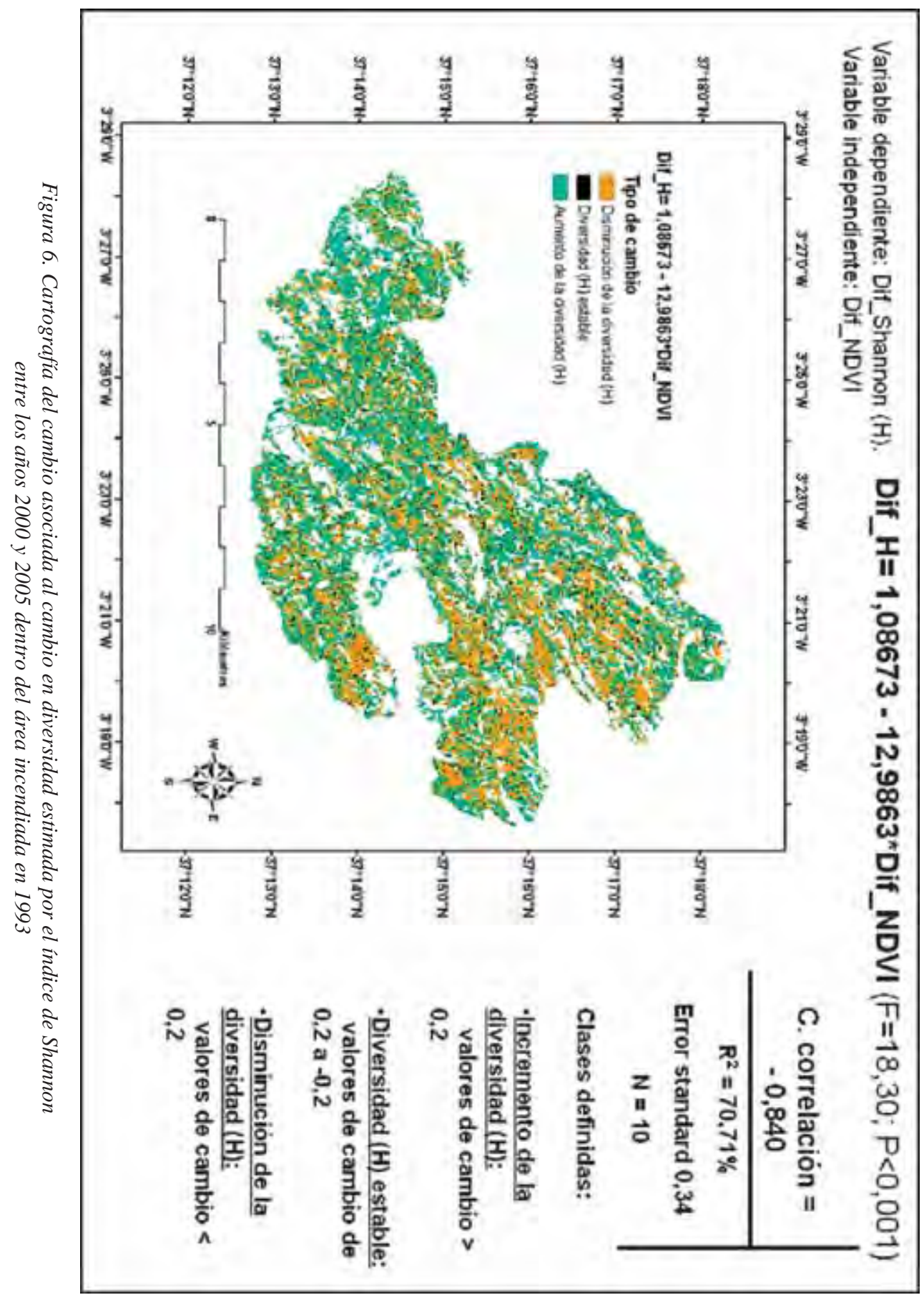




\section{Conclusiones}

La metodología empleada proporcionó resultados fiables y de gran utilidad para los cuatro objetivos que abarca este trabajo: delimitación de la zona afectada, tipificación de la vegetación, análisis de cambio en el grado de cobertura vegetal y evaluación de la dinámica de la diversidad florística. La dinámica de cambio de la diversidad, relacionada con las pautas de regeneración y evolución de la dominancia de las especies colonizadoras dentro del área, es interpretable a través de la información remota y la modelización de dichas variables es fundamental para definir las actuaciones a acometer si se pretende restaurar la cubierta vegetal. La teledetección y los SIG constituyen una herramienta eficaz para la evaluación de la regeneración del grado de cobertura vegetal y el estudio de la dinámica post-incendio de la diversidad y, por tanto, se recomienda su introducción en el manejo y la restauración de espacios naturales.

\section{Agradecimientos}

A todas aquellas personas que proporcionaron información y material de la zona afectada por el incendio: Francisco Pérez-Raya y Joaquín Molero (Universidad de Granada); José María Irurita, José Manuel Moreira y Francisco Bonet (Consejería de Medio Ambiente de la Junta de Andalucía).

\section{Referencias bibliográficas}

Bonham, C.D. (1989). Measurements for terrestrial vegetation. John Wiley and Sons. New York, NY.

Caselles, V. y López García, M.J. (1989). An alternative simple approach to estimate atmospheric correction in multitemporal studies. International Journal of Remote Sensing 10: 1127-1134.

Díaz-Delgado, R. y Pons, X. (2001). Spatial patterns of forest fires in Catalonia (NE Spain) along the period 1975-1995. Analysis of vegetation recovery after fire. Forest Ecology and Management 147: 67-74.

HANES, T.L. (1971). Succession after fire in the chaparral of southern California. Ecological Monographs 41: 27-52.

HenRy, M.C. y Hope, A.S. (1998). Monitoring post-burn recovery of chaparral vegetation in southern California using multi-temporal satellite data. International Journal of Remote Sensing 19: 3097-3107

Heredia laclaustra, A., Martínez Sánchez, S., Quintero, E., Piñeros, W. y ChuVIECO, E. (2003). Comparación de distintas técnicas de análisis digital para la cartografía de áreas quemadas con imágenes LANDSAT ETM+. GeoFocus 3: 216-234. 
Hernández Clemente, R., Navarro Cerrillo, R.M., Gitas, i.A. y Hernández BERMEJO, J.E. (2006). Modelling and monitoring post-fire vegetation recovery and diversity dynamics: a diachronic approach using satellite time-series data set. En $V$ International Conference on Forest Fire Research. ISSN 0378-1127. Coimbra, Portugal.

NAVEH, Z. (1975). The evolutionary significance of fire in the Mediterranean region. Vegetatio 29: 199-208.

PAusAs, J.G. (2004). La recurrencia de incendios en el monte mediterráneo. En Avances en el estudio de la gestión del monte mediterráneo (Vallejo, V.R. y Alloza, J. A. Eds.). CEAM, Valencia, 47-64.

PiUssi, P. (1992). Environmental changes in forest. Examples from the south of Europe. En Response of forest ecosystems to environment changes (Taller, A., Mathy, P. y Jeffers, J.N.R. Eds.). Elsevier Applied Science, London, UK, 298-309.

Riaño, D., Chuvieco, E., Ustin, S., Zomer, R., Dennison, P., Roberts, D. y Salas, J. (2002). Assessment of vegetation regeneration post-fire through multitemporal analysis of AVIRIS images in the Santa Monica Mountains, Remote Sensing of Environment 79: 60-71.

Rivas-Martínez, S., Asensi, A., Díez-Garretas, B., Molero, J. y Valle, F. (1997). Biogeographical synthesis of Andalusia (southern Spain). Journal of Biogeography 24: 915-928.

Salvador, R., Valeriano, J., Pons, X. y Díaz-Delgado, R. (2000). A semiautomatic methodology to detect fire scars in shrubs and evergreen forests with Landsat MSS time series. International Journal of Remote Sensing 21: 655-673.

Siljestrom, P. y Moreno, A. (1995). Monitoring burnt areas by principal components analysis of multitemporal TM data. International Journal of Remote Sensing 16: 1577-1587.

Viedma, O., Meliá, J., Segarra, D. y García-Haro, J. (1997). Modelling rates of ecosystem recovery after fires by using Landsat TM Data. Remote Sensing of Environment 61 : 383-398.

Trabaud, L. y LePart, J. (1980). Floristic changes in Quercus coccifera L. garrigue according to different fires regimes. Vegetatio 46: 05-116. 